\title{
Safety of air travel in the immediate postoperative period after anatomic pulmonary resection
}

\author{
Stephen D. Cassivi, MD, Karlyn E. Pierson, MAN, RN, Bettie J. Lechtenberg, MBA,
}

Francis C. Nichols III, MD, K. Robert Shen, MD, Mark S. Allen, MD, and Dennis A. Wigle, MD, PhD

\section{ABSTRACT}

Objective: The study objective was to determine whether air travel in the immediate postoperative period after anatomic pulmonary resection is associated with increased morbidity or mortality.

Methods: All patients undergoing anatomic pulmonary resection at the Mayo Clinic (2005-2012) were identified and sent surveys querying their mode of transportation home after hospital dismissal and any complications encountered during or shortly after this travel. This included pneumonia, hospital readmission, deep venous thrombosis/pulmonary embolism, and specific pleural complications (pneumothorax, empyema, or chest tube placement). We compared the results of patients returning home by conventional ground travel with the results of patients using air travel.

Results: Surveys were sent to 1833 patients, and 817 responded (44.6\% response rate). A total of 96 responders (11.8\%) used air travel (median distance, $1783 \mathrm{~km}$; range, 486-9684 km) compared with $278 \mathrm{~km}$ (range, 1-2618 km) for the $721 \mathrm{re}$ sponders $(88.2 \%)$ using ground travel $(P<.0001)$. Male patients used air travel more than female patients $(14.4 \%$ vs $9.3 \% ; P=.02)$. Otherwise, no significant differences were observed between the 2 groups. The median duration of hospitalization was 5 days in both groups using air travel (range, 1-25 days) and ground travel (range, $1-42$ days) $(P=.83)$. There was no mortality due to postdismissal travel. The rates of major complication after hospital dismissal for those using air and ground travel were $8.3 \%$ and $7.8 \%$, respectively $(P=.87)$.

Conclusions: Overall major complications are rare in the immediate posthospital dismissal period after lung resection. Air travel during this period was not associated with any significant increase in risk of complications when compared with conventional ground transportation and seems to be a safe option for patients after chest tube removal. (J Thorac Cardiovasc Surg 2017;153:1191-6)

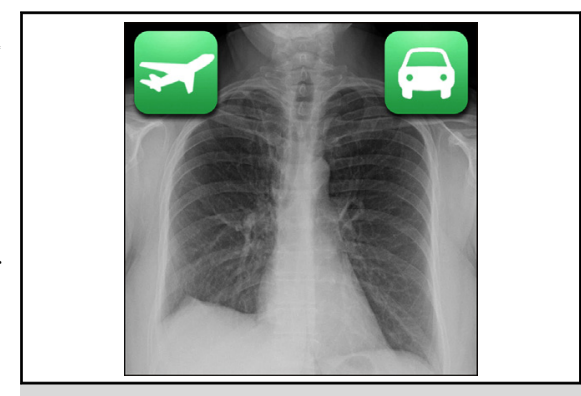

After resection, patients and surgeons face a dilemma regarding options for safe travel.

\section{Central Message}

Air travel after lung resection is not associated with an increased risk of complications when compared with conventional ground transport.

\section{Perspective}

Recommendations regarding the safety and timing of air travel after lung resection have, to date, been based solely on theoretic concerns and expert opinion. The current study provides objective clinical data that show the safety of air travel after lung surgery is equivalent to traveling by conventional ground transportation. The basic criteria for dismissal can be applied similarly to both groups.
Pulmonary resections are the most common major procedure performed by general thoracic surgeons. ${ }^{1}$ The timing of postoperative travel becomes an important consideration given concerns regarding air pressure changes encountered in flight even on modern aircraft with pressurized cabins. ${ }^{2-7}$

\footnotetext{
From the Division of General Thoracic Surgery, Department of Surgery, Mayo Clinic, Rochester, Minn.

Institutional Review Board Approval: Mayo Clinic, IRB\#11-002829, 05/20/2011.

Read at the 94th Annual Meeting of The American Association for Thoracic Surgery, Toronto, Ontario, Canada, April 26-30, 2014.

Received for publication April 28, 2014; revisions received Nov 18, 2016; accepted for publication Dec 17, 2016.

Address for reprints: Dennis A. Wigle, MD, PhD, Mayo Clinic, 200 First St SW,

Rochester, MN 55905 (E-mail: wigle.dennis@mayo.edu).

0022-5223/\$36.00

Copyright (c) 2017 by The American Association for Thoracic Surgery

http://dx.doi.org/10.1016/j.jtcvs.2016.12.035
}

The most recent guidelines from the Aerospace Medical Association state that "pneumothorax is an absolute contraindication to air travel" and recommend air travel be delayed by 2 to 3 weeks after uncomplicated thoracic surgery. ${ }^{8}$ However, patients increasingly are traveling long distances to obtain specialty care, including general thoracic surgical procedures. Patients often choose to travel these long distances by airplane with the

Scanning this QR code will take you to the appendix for this article.

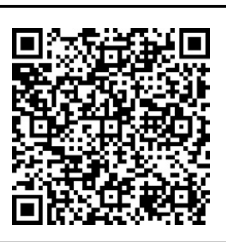




\section{Abbreviations and Acronyms \\ $\mathrm{CI}=$ confidence interval \\ $\% \mathrm{DLCO}=$ percent predicted of diffusing capacity of the lungs for carbon monoxide \\ $\% \mathrm{FEV}_{1}=$ percent predicted of forced expiratory volume in the first second \\ $\mathrm{OR}=$ odds ratio \\ $\% \mathrm{RV} \quad=$ percent predicted of residual volume}

hope of returning home promptly again by air travel after surgery.

It has been the practice at the Mayo Clinic to allow prompt return home after thoracic surgical procedures and dismissal from the hospital regardless of the mode of return transport, be it by ground or air travel. We sought to retrospectively examine a recent cohort of patients undergoing anatomic pulmonary resection to compare the outcomes according to their mode of return transport after dismissal from the hospital.

\section{MATERIALS AND METHODS}

Institutional review board approval was obtained a priori for this study, and only patients who had provided consent for inclusion in clinical studies were included in this investigation. We identified all patients undergoing anatomic pulmonary resection at the Mayo Clinic (Rochester, Minn) from 2005 to 2012 from a prospectively collected clinical database. Patients who died during their initial postoperative hospitalization were excluded, as were patients who were known to have died 30 days or more after hospital dismissal. We also investigated the cases of any patients who died within 30 days of dismissal from the hospital for cause of death to ascertain whether this was related to the mode of return transport.

All remaining patients, those assumed to be still alive since surgery, were sent survey questionnaires querying their mode of transportation after hospital dismissal by air or ground travel. The questionnaire also requested details regarding any complications encountered during or shortly after this travel, such as pneumonia, hospital readmission, deep venous thrombosis, or pulmonary embolism. It also asked about specific pleural complications, such as pneumothorax, empyema, or the requirement for further pleural drainage (Appendix E1). Returned questionnaires were tabulated and compared with records from the first postoperative visit. Equivocal responses were followed up by telephone interview. We also collected demographic data from the medical records, including age, sex, and distance traveled after hospital dismissal, as well as clinical data, including the percent predicted of forced expiratory volume in the first second $(\%$ $\mathrm{FEV}_{1}$ ), percent predicted of diffusing capacity of the lungs for carbon monoxide $(\% \mathrm{DLCO})$, percent residual volume $(\% \mathrm{RV})$, surgical approach (open or video-assisted thoracic surgery), type of resection, and pertinent characteristics of the last chest radiograph obtained before hospital dismissal.

Descriptive statistics are reported as mean and standard deviation for continuous variables and as number (percentage) for discrete variables. Patient characteristics were compared between patients traveling by air and patients traveling by ground using a 2 -sample $t$ test for continuous variables and a chi-square test for discrete variables. Possible survey response bias was examined looking at the association of baseline patient characteristics with survey completion using logistic regression and response to the survey as the dependent variable in these models.
The association of mode of patient travel (air relative to ground) with the odds of a postdismissal major complication was assessed using logistic regression. Results are reported as odds ratio (OR) and $95 \%$ confidence interval (CI). Likewise, other patient characteristics were assessed for an association with a postdismissal major complication.

By using backward selection, a multiple variable model was identified to examine the association of transportation mode, sex, and $\% \mathrm{FEV}_{1}$ with the odds of a postdismissal major complication. Two variables, \%DLCO and $\% \mathrm{RV}$, were not considered for inclusion in the multiple variable model because both variables were missing in more than $10 \%$ of the study patients. The alpha level was set at 0.05 for statistical significance.

We evaluated the data obtained to determine the rate of postoperative mortality and morbidity and used univariate and multivariable analyses to determine the factors associated with an increased risk of postoperative mortality and morbidity.

\section{RESULTS}

During the study period, 2494 patients underwent anatomic pulmonary resection (Figure 1). A total of 661 patients $(26.5 \%)$ died before the survey. Twelve patients $(0.5 \%)$ died within 30 days after dismissal from hospital. None of these 12 patients used air travel after dismissal from hospital, and in none of the cases was death related to postdismissal travel.

Survey questionnaires were sent to 1833 patients. A total of 817 patients $(44.6 \%)$ returned surveys and are included in the subsequent analysis. To assess for response bias, we compared the demographic characteristics of responders with those of nonresponders. Responders were older (65.6 vs 63.2 years; $P<.0001$ ) and more likely to have undergone a video-assisted thoracic surgery lung resection $(18.3 \%$ vs $13.4 \% ; P=.004)$ than nonresponders. The distance from the Mayo Clinic to the responder's home was greater than for nonresponders ( $615 \mathrm{vs} 415 \mathrm{~km} ; P<.0001)$. Otherwise, there were no significant differences between the survey responders and eligible patients who did not respond to the survey.

Of the survey respondents, $721(88.2 \%)$ traveled by ground and $96(11.8 \%)$ traveled by air. In those traveling by air, $83(86.5 \%)$ used commercial airlines and 13 $(13.5 \%)$ returned home by private jet. Furthermore, the number of flights taken (segments of air travel) to return home was 1 for 50 patients $(52.6 \%), 2$ for 38 patients $(40.0 \%)$, and 3 for 7 patients $(7.4 \%)$; 2 patients $(2.4 \%)$ did not report this information. The median distance to return home was $1783 \mathrm{~km}$ (range, 486-9684 km) for those traveling by air and $278 \mathrm{~km}$ (range, $1-2618 \mathrm{~km}$ ) for those traveling by ground $(P<.0001)$.

The patient demographic and clinical characteristics are detailed in Table 1 . There were only 2 significant differences between those traveling by air and ground. There was a higher proportion of men traveling by air compared with those traveling by ground $(58.3 \%$ vs $46.0 \%$, respectively; $P<.024)$. Second, and predictably, those traveling by air traveled farther than those traveling by ground (median distance: 1783 vs $278 \mathrm{~km}$, respectively; 


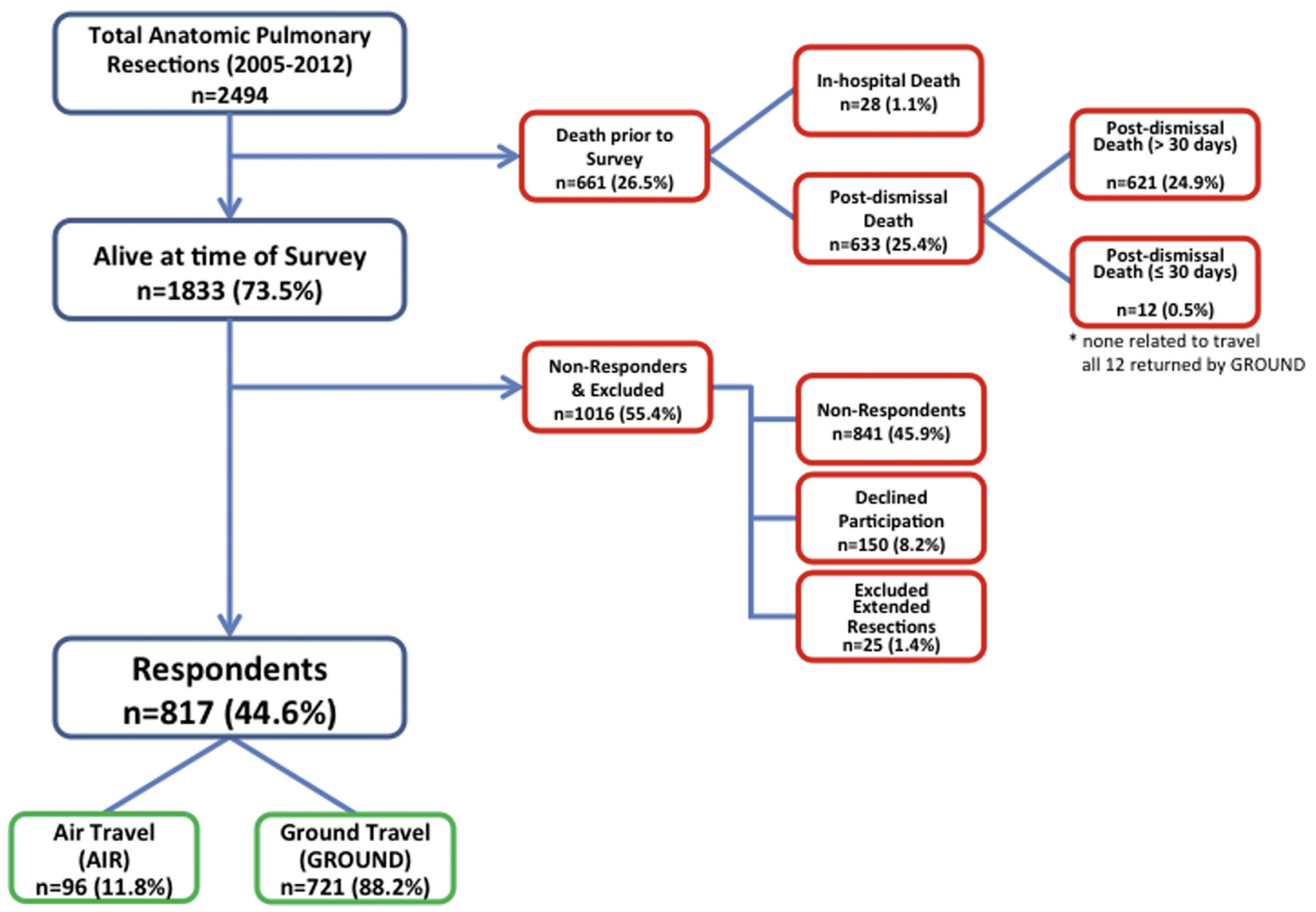

FIGURE 1. Patient identification and accrual chart.

$P<.0001)$. There were no statistically significant differences in age, $\% \mathrm{FEV}_{1}, \% \mathrm{DLCO}, \% \mathrm{RV}$, thoracoscopic or open approaches, extent of resections, predismissal chest radiograph characteristics, or length of stay. The interval between the predismissal chest radiograph and hospital dismissal was neither clinically nor statistically different at 1.2 days and 1.0 day for those traveling by air and ground, respectively, although the $P$ value was .09 . Specifically, $25 \%$ and $37 \%$ of patients traveling by air and ground, respectively, were sent home on the same day as their final chest radiograph, whereas $46 \%$ (air travel) and $42 \%$ (ground travel) were dismissed from the hospital on the day after their predismissal chest radiograph. Furthermore, in those traveling by air, the median interval from hospital dismissal to air travel was 2 days (range, $0-45$ days). We noted that 37 respondents $(4.5 \%)$ were dismissed from the hospital with a chest tube. All of these patients had their chest tube connected to a Heimlich 1-way valve, and all were traveling by ground. With regard to the pleural gap seen in patients with a pneumothorax on their chest radiograph before dismissal, the range for patients in the pleural gap greater than $1 \mathrm{~cm}$ category was 1 to $4 \mathrm{~cm}$, with most being less than 2 to $3 \mathrm{~cm}$.
There were 64 postdismissal major complications $(7.8 \%)$ reported overall in the study cohort. Eight $(8.3 \%)$ occurred in those traveling by air, and $56(7.8 \%)$ occurred in those traveling by ground; there was no statistically significant difference between the 2 groups $(P=.872)$. By using the observed sample size in a post hoc power calculation, there was $80 \%$ power to detect an OR of 2.5 or greater.

Major complications reported included pneumonia, empyema, deep venous thrombosis, pulmonary embolism, severe dyspnea, chest tube site drainage, pneumothorax, severe pain, and major cardiac arrhythmia (Table 2). Rehospitalization occurred in 90 patients $(11.0 \%)$ in the overall cohort, with $12(12.5 \%)$ in those traveling by air and 78 $(10.8 \%)$ in those traveling by ground $(P=.648)$. A chest tube was reinserted in 19 patients $(2.3 \%)$, with $3(3.1 \%)$ in those traveling by air and $16(2.2 \%)$ in those graveling by ground $(P=.582)$. Of note, there was no overlap between the patients describing severe dyspnea and the patients who developed a pneumothorax after hospital dismissal.

Univariate analysis was performed to assess for risk factors associated with postdismissal major complications (not shown). Air travel to return home postdismissal was not associated with a significantly increased 
TABLE 1. Patient characteristics

\begin{tabular}{|c|c|c|c|c|}
\hline Patient characteristics & Air travel $(n=96)$ & Ground travel $(n=721)$ & Total $(\mathbf{n}=\mathbf{8 1 7})$ & $P$ value \\
\hline \multicolumn{5}{|l|}{ Age, y } \\
\hline Mean \pm SD & $69.7 \pm 10.7$ & $69.2 \pm 11.7$ & $69.3 \pm 11.6$ & .9875 \\
\hline Sex & & & & .0235 \\
\hline Female & $40(41.7 \%)$ & $389(54.0 \%)$ & $429(52.5 \%)$ & \\
\hline Male & $56(58.3 \%)$ & $332(46.0 \%)$ & $388(47.5 \%)$ & \\
\hline \multicolumn{5}{|l|}{$\% \mathrm{FEV}_{1}$} \\
\hline Mean $\pm \mathrm{SD}$ & $80 \% \pm 20 \%$ & $80 \% \pm 20 \%$ & $80 \% \pm 20 \%$ & .8937 \\
\hline \multicolumn{5}{|l|}{$\%$ DLCO } \\
\hline Mean \pm SD & $80 \% \pm 20 \%$ & $80 \% \pm 20 \%$ & $80 \% \pm 20 \%$ & .6161 \\
\hline \multicolumn{5}{|l|}{$\% \mathrm{RV}$} \\
\hline Mean \pm SD & $130 \% \pm 40 \%$ & $140 \% \pm 40 \%$ & $140 \% \pm 40 \%$ & .5132 \\
\hline Surgical approach & & & & .7504 \\
\hline Open & $77(80.2 \%)$ & $588(81.6 \%)$ & $665(81.4 \%)$ & \\
\hline VATS & $19(19.8 \%)$ & $133(18.4 \%)$ & $152(18.6 \%)$ & \\
\hline Type of resection & & & & .8776 \\
\hline Segmentectomy (anatomic) & $13(13.5 \%)$ & $106(14.7 \%)$ & $119(14.6 \%)$ & \\
\hline Lobectomy & $75(78.1 \%)$ & $550(76.3 \%)$ & $625(76.5 \%)$ & \\
\hline Bilobectomy & $2(2.1 \%)$ & $25(3.5 \%)$ & $27(3.3 \%)$ & \\
\hline Pneumonectomy & $6(6.3 \%)$ & $40(5.5 \%)$ & $46(5.6 \%)$ & \\
\hline Predismissal chest $\mathrm{x}$-ray result & & & & .9863 \\
\hline Normal & $18(18.8 \%)$ & $152(21.1 \%)$ & $170(20.8 \%)$ & \\
\hline SE only & $14(14.6 \%)$ & $98(13.6 \%)$ & $112(13.7 \%)$ & \\
\hline Pleural gap $\leq 1 \mathrm{~cm}$ only & $18(18.8 \%)$ & $138(19.1 \%)$ & $156(19.1 \%)$ & \\
\hline Pleural gap $>1 \mathrm{~cm}$ only & $23(24.0 \%)$ & $153(21.2 \%)$ & $176(21.5 \%)$ & \\
\hline $\mathrm{SE}+$ pleural gap $\leq 1 \mathrm{~cm}$ only & $13(13.5 \%)$ & $99(13.7 \%)$ & $112(13.7 \%)$ & \\
\hline $\mathrm{SE}+$ pleural gap $>1 \mathrm{~cm}$ only & $10(10.4 \%)$ & $81(11.2 \%)$ & $91(11.1 \%)$ & \\
\hline \multicolumn{5}{|l|}{ Length of stay (d) } \\
\hline Mean \pm SD & $5.8 \pm 3.4$ & $5.9 \pm 3.9$ & $5.9 \pm 3.8$ & .83 \\
\hline \multicolumn{5}{|c|}{$\begin{array}{l}\text { Interval from predismissal chest } \mathrm{x} \text {-ray } \\
\text { to dismissal (d) }\end{array}$} \\
\hline Median, IQR & $1.0,0-2$ & $1.0,0-1$ & $1.0 \pm 1.3$ & .02 \\
\hline \multicolumn{5}{|l|}{ Postoperative return distance $(\mathrm{km})$} \\
\hline Median, IQR & $1783,1044-2533$ & $278,137-561$ & $370,146-720$ & $<.0001$ \\
\hline
\end{tabular}

$S D$, Standard deviation; $\% F E V_{l}$, percent predicted of forced expiratory volume in the first second; $\% D L C O$, percent predicted of diffusing capacity of the lungs for carbon monoxide; $\% R V$, percent predicted of residual volume; VATS, video-assisted thoracic surgery; $S E$, subcutaneous emphysema; $I Q R$, interquartile range.

risk of postdismissal major complications with an OR of $1.08(95 \%$ CI, 0.50-2.34; $P=.846)$. Female sex (OR, $0.55 ; P=.027), \% \mathrm{FEV}_{1}(\mathrm{OR}, 0.84$ per $10 \%$ increment; $P=.007)$, and $\%$ DLCO (OR, 0.85 per $10 \%$ increment;
$P=.037)$ showed a statistically significant association with major complications after hospital discharge.

In multiple variable model including sex and $\% \mathrm{FEV}_{1}$, there was again no significant association of a patient

TABLE 2. Major complications

\begin{tabular}{|c|c|c|c|c|}
\hline & Air travel $(n=96)$ & Ground travel $(n=721)$ & Total $(\mathbf{n}=\mathbf{8 1 7})$ & $P$ value \\
\hline Total patients $(\%)$ & $8(8.3 \%)$ & $56(7.8 \%)$ & $64(7.8 \%)$ & .872 \\
\hline \multicolumn{5}{|l|}{ Complication } \\
\hline Pneumonia/empyema & $3(3.1 \%)$ & $29(4.0 \%)$ & $32(3.9 \%)$ & 1.0 \\
\hline DVT/PE & $1(1.0 \%)$ & $10(1.4 \%)$ & $11(1.3 \%)$ & 1.0 \\
\hline Severe dyspnea & $2(2.1 \%)$ & $6(0.8 \%)$ & $8(1.0 \%)$ & .240 \\
\hline Chest tube site drainage & $0(0.0 \%)$ & $5(0.7 \%)$ & $5(1.0 \%)$ & .846 \\
\hline Pneumothorax & $2(2.1 \%)$ & $2(0.3 \%)$ & $4(0.5 \%)$ & .070 \\
\hline Severe pain & $0(0.0 \%)$ & $2(0.3 \%)$ & $2(0.2 \%)$ & 1.0 \\
\hline Major cardiac arrhythmia & $0(0.0 \%)$ & $1(0.1 \%)$ & $1(0.1 \%)$ & 1.0 \\
\hline Other & $0(0.0 \%)$ & $1(0.1 \%)$ & $1(0.1 \%)$ & 1.0 \\
\hline Rehospitalization & $12(12.5 \%)$ & $78(10.8 \%)$ & $90(11.0 \%)$ & .648 \\
\hline Chest tube replacement & $3(3.1 \%)$ & $16(2.2 \%)$ & $19(2.3 \%)$ & .582 \\
\hline
\end{tabular}

$D V T$, Deep venous thrombosis; $P E$, pulmonary embolism. 
traveling by air and an increased risk of a postdismissal major complication (OR, $1.1 ; 95 \% \mathrm{CI}, 0.5-2.5 ; P=.78)$.

\section{DISCUSSION}

Current safety guidelines regarding air travel after lung surgery remain limited to ad hoc recommendations from individual commercial airlines and the more formal Aerospace Medical Association medical guidelines for air travel published in $2003 .{ }^{8}$ Airline industry recommendations are varied but are, in general, fairly conservative. The Aerospace Medical Association guidelines cover a wide spectrum of issues related to aviation medicine with only a small section dealing directly with air travel after thoracic surgery or after experiencing a pneumothorax. In making the explicit statement that "pneumothorax is an absolute contraindication to air travel" and advocating for an interval of 2 to 3 weeks before air travel after thoracic surgery, the Association's guidelines task force has based its recommendations on expert opinion given the lack of published research in this field.

A wide variability of recommendations are currently seen within the clinical practice of thoracic surgeons. A recent survey of thoracic surgeons showed a high variability in their recommendations for travel after thoracic surgery. Respondents to this survey reported advising patients with a postoperative pneumothorax to avoid air travel for an average of 12 days, with the range of responses being 0 to 60 days. ${ }^{6}$ Furthermore, $53 \%$ of respondents required resolution of any pneumothorax before allowing air travel in their postoperative patients.

The potential concerns regarding air travel after thoracic surgery relate to 3 main issues. First, for most flights, the actual cruising altitude ranges between 7000 and $14,000 \mathrm{~m} \mathrm{(20,000}$ and 40,000 ft). With pressurization, the airplane cabin becomes a hypobaric milieu during flight with the equivalent altitude range being 1500 to $2500 \mathrm{~m}$ (5000-8000 ft) above sea level. In accordance with Boyle's law, when air pressure is lowered, gases expand. This raises the concern about increasing the size and effect of a preexisting pneumothorax and subcutaneous emphysema. Second, the decreased cabin air pressure diminishes the partial pressure of oxygen (Dalton's law) and therefore can exacerbate hypoxemia. Third, there are concerns related to dehydration potentiating the risk of postoperative deep venous thrombosis because of low relative humidity in the airplane cabin. ${ }^{2}$

In our study, we did not find a difference in overall major complications, rehospitalization, or need for chest tube placement for patients who returned home by air or ground transportation. Of note, the 2 groups were similar in all regards except that there was a higher proportion of men traveling by air and that those traveling by air traveled farther to return home after hospital dismissal. Specifically, there did not seem to be a significant difference in dismissal management for the 2 groups because there was no difference between those traveling by air and those traveling by ground in the overall length of stay or the interval from the predismissal chest radiograph to dismissal.

When looking at the specific major complications, there were no statistically significant differences between those traveling by air and ground. Nevertheless, it is worth noting that for pneumothorax and severe dyspnea, we observed a slight but not significant increased rate in those traveling by air. Our analysis also showed that the only factors associated with a statistically significant increased risk of major complications after hospital dismissal were male sex, decreasing $\% \mathrm{FEV}_{1}$, and decreasing $\%$ DLCO. Of note, the extent of resection, the surgical approach, and the predismissal chest radiograph result were not associated with differences in postdismissal outcomes.

\section{Study Limitations}

Although limited by its retrospective nature, the favorable response rate of our study $(44.6 \%)$ does provide us with a suitable cohort of patients to evaluate. The analysis of the data shows that the rate of major complications $(8 \%)$ and rehospitalization $(11 \%)$ in the immediate posthospital dismissal period is relatively low. Furthermore, air travel during this period was not associated with any significant increase in risk of complications when compared with conventional ground transportation. We also acknowledge a number of further limitations to our study. We relied on patient-reported outcomes during the perioperative period, which in some instances was up to 10 years previously. Also of note, this study does not apply to patients with a chest tube and Heimlich valve in place at the time of air travel, because none of the patients included in the study underwent these conditions. Patients who were deceased at the time of the study were not included, and it is possible that some patients developed a postoperative or travel-related complication that led to death more than 30 days after discharge.

\section{CONCLUSIONS}

These findings support our current practice of allowing patients to return home by air travel after pulmonary resection using the same criteria that is used for patients who are traveling by ground transportation. These include chest tube removal after absence of ongoing air leak and output less than $300 \mathrm{~mL}$ over 24 hours, adequate pain management with oral analgesia, an active ambulation schedule evaluated and monitored by the unit-based respiratory therapist, and evaluation for our postoperative inpatient pulmonary rehabilitation program that they receive on the nursing unit if appropriate.

\section{Conflict of Interest Statement}

Authors have nothing to disclose with regard to commercial support. 
The authors acknowledge the expert statistical assistance of W. Scott Harmsen, PhD, of the Mayo Clinic Department of Biostatistics, Kristin A. Holst, for expert assistance in manuscript preparation, and Clayton T. Cowl, MD, of the Mayo Clinic Division of Aerospace Medicine, for expert advice and analysis.

\section{References}

1. Kozower BD, Sheng S, O'Brien SM, Liptay MJ, Lau CL, Jones DR, et al. STS database risk models: predictors of mortality and major morbidity for lung cancer resection. Ann Thorac Surg. 2010;90:875-81.

2. Skjenna OW, Evans JF, Moore MS, Thibault C, Tucker AG. Helping patients travel by air. CMAJ. 1991;144:287-93.

3. Gong H Jr. Advising patients with pulmonary diseases on air travel. Ann Intern Med. 1989;111:349-51.
4. Schwartz JS, Bercowitz HZ, Moser KM. Air travel hypoxemia with chronic obstructive pulmonary disease. Ann Intern Med. 1984;100:473-7.

5. Dillard TA, Berg BW, Rajagopal KR, Dooley JW, Mehm WJ. Hypoxemia during air travel in patients with chronic obstructive pulmonary disease. Ann Intern Med. 1989;111:362-7.

6. Szymanski TJ, Jaklitsch MT, Jacobson F, Mullen GJ, Ferrigno M. Expansion of postoperative pneumothorax and pneumomediastinum: determining when it is safe to fly. Aviat Space Environ Med. 2010;81:423-6.

7. Sacco F, Calero KR. Safety of early air travel after treatment of traumatic pneumothorax. Int J Circumpolar Health. 2014;73:1-3.

8. Aerospace Medical Association Medical Guidelines Task Force. Medical guidelines for air travel (2nd edition). Aviat Space Environ Med. 2003;74: A1-19.

Key Words: air travel, flying, lung surgery, pneumothorax, safety 


\section{APPENDIX E1. QUESTIONNAIRE ASSESSING THE SAFETY OF FLYING AFTER A LOBECTOMY}

1. Did you fly home? Yes __ No (If no, skip to question 2)

a. How many days after discharge did you leave Rochester?

b. Did you fly: __Commercial __ Private jet

c. How many segments (involving a stopover, change of aircraft, or change of airline) were in your flight?

2. Did you experience any health problems with your travel back home?

_ Yes _ No

3. If yes, please select all that apply from the list below: Nonpulmonary (not related to your lungs)

Pulmonary (related to your lungs)

Shortness of breath

Needed oxygen

Hospitalized

Other minor, please specify:

Other major, please specify:

*The following questions relate to your recovery between hospital discharge and your follow-up visit with your care provider (typically 4 to 6 weeks following surgery).

4. Did you get pneumonia?

Y Yes _ No

5. Did you have any chest drainage from your incision?

Yes __ No

6. If you did have chest drainage from your incision, was the drainage: _Significant __ Minor

7. Did you get subcutaneous emphysema or air under the skin?

Y Yes _ No

8. Did you get re-hospitalized, between discharge and your follow-up visit?

Yes No

9. If you were re-hospitalized, what was the reason?

10. Did you get another chest tube?

Yes _ No

11. Did you have any other complications?

_Yes _No

12. If you did have other complications, what were they? 